Pacific Journal of Mathematics

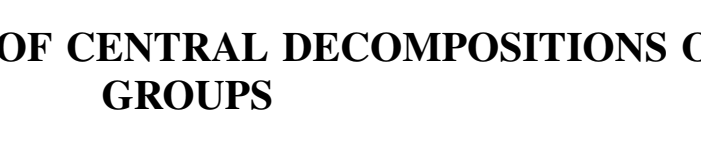




\title{
ON UNIQUENESS OF CENTRAL DECOMPOSITIONS OF GROUPS
}

\author{
C. Y. TANG
}

In this paper a condition for two central decompositions of a group into centrally indecomposable factors to be isomorphic modulo the center is derived. Using this result a larger class of groups with central decompositions into centrally indecomposable factors isomorphic modulo the center than that of an earlier work of the author is determined. An example of a group having two central decompositions into centrally indecomposable factors which are not isomorphic modulo the center is also obtained.

The purpose of this paper is to extend the results of an earlier work of the author [5]. The central products defined in [5] are special cases of the generalized direct products in [4]. The term 'central product' was introduced by P. Hall in [2]. In [1] Gorenstein discussed the irreducible representations of central products in terms of those of the individual factors and also made use of them in the study of extra-special $p$-groups and critical subgroups of $p$-groups. It is of interest to note that in these cases the central products involved all have central factors whose factor groups with respect to their centers are abelian of rank $\leqq 3$. The results of this paper show that all such central factors are centrally indecomposable and moreover any two such decompositions are isomorphic modulo the center. It must, however, be noted that in [1] central products are used in a more general sense, in that the amalgamated subgroup is allowed to be a proper subgroup of the center, while in our case the amalgamated subgroup must be the center itself. But this does not affect the above observation.

All notations and terminology will be the same as in [5], and unless otherwise specified $H$ shall always mean $Z(G)$. Moreover, the ascending and descending chain conditions for normal subgroups are always assumed and the rank of an abelian group is used in the sense of Prüfer (Kaplansky [3]). Thus $G=(A \times B)_{I I}$ means that $G=A B$, with $[A, B]=1$ and $A \cap B=H=Z(G)$. Two central decompositions of $G$ are said to be isomorphic modulo $H$ if the induced direct decompositions of $G / H$ are isomorphic. Our problem is to find conditions for a group to have unique, up to isomorphism modulo $H$, central decomposition into centrally indecomposable factors.

The following results from [5] will be needed: 
Lemma 1. (Lemma 3.1, [5]). If $G=(A \times B)_{H}=(C \times D)_{H}$ and $x \in C$ then there exist $a \in A$ and $b \in B$ with $a$ and $b$ respectively of the form $a=c d$ and $b=c^{K} d^{-1}$ where $c, c^{*} \in C$ and $d \in D$ such that $x=a b$.

A sharper form of this result will be:

Lemma 1a. If $G=(A \times B)_{I I}=(C \times D)_{H}$ and $x \in C$ then there exist $c \in C$ and $d \in D$ such that $c d \in A$ and $c^{-1} x d^{-1} \in B$.

Proof. Let $x=a b$ with $a \in A$ and $b \in B$. Then $a=c d$ and $b=$ $c^{\prime} d^{\prime}$ for some $c, c^{\prime} \in C$ and $d, d^{\prime} \in D$. Thus $x=c d c^{\prime} d^{\prime}=c c^{\prime} d d^{\prime}$. Hence $d d^{\prime} \in C \cap D=H$. Therefore $d^{\prime}=d^{-1} h$ for some $h \in H$, whence $\left[d, d^{\prime}\right]=$ 1. It follows that $c^{-1} x d^{-1}=c^{\prime} d^{\prime}$.

Lemma 1 follows from Lemma 1a by putting $c^{*}=c^{-1} x$.

Lemma 2. (Lemma 3.4, [5]). If $G=(A \times B)_{H}=(C \times D)_{H}$ and $M$ is a subgroup of $A$ containing $H$ such that $M \subset P_{A}(C)$ and $C=$ $P_{C}(M)$, then $A=(M \times N)_{I I}$ where $N=A \cap D$.

$P_{A}(C)$ and $P_{C}(M)$ are respectively the $H$-projections of $C$ in $A$ and $M$ in $C$. In general if $G=\left(\prod_{i=1}^{n} G_{i}\right)_{I I}$ and $\theta$ is the homomorphism mapping $G$ onto $\bar{G}=G / H$ then the $H$-projection of $x \in G$ in $G_{i}$ is defined to be the set of all preimages of $(x \theta) \theta_{i}$ under $\theta$, where $\theta_{i}$ is the $\bar{G}_{i}$-decomposition operator of $\bar{G}=\prod_{i=1}^{n} \bar{G}_{i}, \bar{G}_{i}=G_{i} \theta$. This set is denoted by $P_{G_{i}}(x)$. Also, for $x \in G, \bar{x}$ shall always mean $x \theta$.

Theorem 1. (Theorem 3.10, [5]). Let $G$ be a nilpotent group of class 2 with a cyclic commutator subgroup. $G$ is centrally indecomposable if and only if $G / H$ is of rank 2 and $C(G)$ is either a p-group or an infinite group.

2. In the theory of direct decompositions it is known that if a group $G$ has a trivial center then $G$ has a unique Remak decomposition. An analogue to this property in the case of central decompositions will be that if the factor group $G / Z(G)$ has a trivial center then the central decomposition of $G$ into centrally indecomposable factors is unique.

The following lemma can be proved by an easy computation:

Lemma 2.1. If $G=(A \times B)_{H}=(C \times D)_{H}$ then $\left[P_{A}(C), P_{A}(D)\right] \subseteq H$.

Lemma 2.2. Let $G=(A \times B)_{H}=(C \times D)_{H}$. If $A \subset C$ then $D \subset B$.

Proof. Assume $A \subset C$. Let $x \in D$. Then $x=a b$ with $a \in A$ and 
$b \in B$. Now $[A, x] \subset[C, D]=1$ and $[A, b] \subset[A, B]=1$. Therefore $[A, a]=1$. Thus $a \in Z(A)=H \subset B$, whence $x=a b \in B$.

\section{CorollaRy 2.3. If $G=(A \times B)_{H}=(A \times D)_{H}$ then $B=D$.}

It may be noted that Corollary 2.3 can also be obtained by applying Theorem 2.5 of [5].

THEOREM 2.4. If $G / H$ has a trivial center, then the central decomposition of $G$ into centrally indecomposable factors is unique.

Proof. Let $G=\left(\prod_{i=1}^{n} G_{i}\right)_{H}=\left(\prod_{i=1}^{m} F_{i}\right)_{H}$ be any two central decompositions of $G$ into centrally indecomposable factors. Let $P=$ $P_{G_{1}}\left(F_{1}\right)$ and $Q=P_{G_{1}}\left(F_{1}^{\prime}\right)$. (Throughout this paper we shall use $G_{i}^{\prime}$ to denote $\left.\left(\prod_{k \neq i}^{n} G_{k}\right)_{H}\right)$. Clearly $G_{1}=P Q$. Indeed we can assume, without loss, that $H \varsubsetneqq P$. Let $x \in G_{1}$. By Lemma 1 , there exist $a \in P, b \in Q$ and $u \in G_{1}^{\prime}$ such that $x=a b$ with $a u \in F_{1}$ and $b u^{-1} \in F_{1}^{\prime}$. Thus $u \in$ $P_{G_{1}^{\prime}}\left(F_{1}\right)$ and $\left.u \in P_{G_{1}^{\prime}}\left(F_{1}^{\prime}\right)\right)$. But by Lemma 2.1, $\left[P_{G_{1}^{\prime}}\left(F_{1}\right), P_{G_{1}^{\prime}}\left(F_{1}^{\prime}\right)\right] \subset H$. Since $G_{1}^{\prime}=P_{G_{1}^{\prime}}\left(F_{1}\right) \cdot P_{G_{1}^{\prime}}\left(F_{1}^{\prime}\right)$, it follows that $\left[u, G_{1}^{\prime}\right] \subset H$, whence $\bar{u} \epsilon$ $Z(G / H)=1, \bar{u}$ being the image of $u$ in $G / H$. Thus $u \in H$. It follows that $a u \in P$ and $b u^{-1} \in Q$. Let $A=P \cap F_{1}$ and $B=Q \cap F_{1}^{\prime}$. It is clear that $G_{1}=(A \times B)_{H}$. But $G_{1}$ is centrally indecomposable. Hence either $A=G_{1}$ and $B=H$ or $A=H$ and $B=G_{1}$. Now $B=G_{1}$ implies that. $Q=G_{1}$. Since by Lemma 2.1, $[P, Q] \subset H$, we have $P / H \subset Z(G / H)=1$. Thus $P \subseteq H$, contradicting the assumption $H \cong P_{G_{1}}\left(F_{1}\right)$. Therefore we must have $A=G_{1}$ and $B=H$. This implies that $P=G_{1}$ and $Q=$ $H$, whence $b \in H$. Thus by Lemma 2 and the indecomposability of $F_{1}$ we have $G_{1}=F_{1}$. Now by Corollary $2.3, G_{1}^{\prime}=F_{1}^{\prime}$. Therefore by induction we have $F_{i}=G_{i}$ after suitable reindexing.

3. From Theorem 1 it follows immediately that if $G$ is a $p$-group of class 2 such that $G / Z(G)$ is of rank 2 then $G$ is centrally indecomposable. In fact in the case of $p$-groups this property can also easily be proved by considering the ranks of abelian groups. Indeed it is not difficult to prove the central indecomposability of a $p$-group $G$ of class 2 with $G / Z(G)$ abelian of rank 3. M. Schick pointed out that any finite $p$-group which can be generated by three of less $H$-generators (in the sense defined below) is centrally indecomposable.

DeFinition 3.1. Let $G$ be any group. If the elements $a_{1}, \cdots, a_{n}$ together with $H$ generate $G$, then $G$ is said to be generated by the $H$-generators $a_{1}, \cdots, a_{n}$.

THEOREM 3.2. If $G$ is a finite p-group with $H=Z(G)$ such that 
$G$ can be generated by three or fewer $H$-generators, then $G$ is centrally indecomposable.

Proof. Suppose $G=(A \times B)_{H}$. We shall show that either $A$ or $B$ is $H$. Let $\psi$ be the canonical homomorphism of $G$ onto $G / N$ where $N=\{\Phi(G), H\}$. Now $G \psi=A \psi \times B \psi$ is elementary abelian and $G \psi$ has rank at most 3. It follows that one of $A \psi$ and $B \psi$ has rank at most 1. If, say, $A \psi=(A / H) / \Phi(A / H)$ is cyclic then $A / H$ is cyclic. This implies that $A$ is abelian whence $A=Z(A)=H$.

4. In this section we shall derive a condition for central decompositions of $G$ into centrally indecomposable factors to be isomorphic modulo the center and apply it to central decompositions of nilpotent groups of class 2 in which the factor group of each factor with respect to the center is abelian of rank $\leqq 3$.

The following lemma can be easily proved.

Lemma 4.1. Let $G=A \times B=C \times D . A$ and $C$ are exchangeable if and only if there exist subgroups $U \subset B$ and $V \subset D$ such that $A \times U=C \times V$ in which $A$ and $C$ are exchangeable.

Lemma 4.2. Let $G=(A \times B)_{H}=(C \times D)_{H}$. If there exist subgroups $H \subset U \subset B$ and $H \subset V \subset D$ such that $(A \times U)_{H}=(C \times V)_{H}$ and $\bar{A}$ and $\bar{V}$ are exchangeable in $\bar{A} \times \bar{U}=\bar{C} \times \bar{V}$, then $G=(A \times U \times$ $B \cap D)_{H}=(C \times V \times B \cap D)_{H}$.

Proof. Since $\bar{A}$ and $\bar{V}$ are exchangeable in $\bar{A} \times \bar{U}=\bar{C} \times \bar{V}$ and $\bar{V} \subset \bar{D}$, therefore $\bar{V} \theta_{A}=\bar{A}$ and $\bar{V} \cong \bar{A} \theta_{D}$ where $\theta_{A}$ is the $\bar{A}$-decomposition operator in $\bar{G}=\bar{A} \times \bar{B}$ and $\theta_{D}$ is the $\bar{D}$-decomposition operator in $\bar{G}=\bar{C} \times \bar{D}$. Thus $P_{A}(V)=A$ and $V \subseteq P_{D}(A)$. Hence by Lemma 2 we have $G=(C \times V \times B \cap D)_{H}$. In the same way we can show that $G=(A \times U \times B \cap D)_{H}$.

THEOREM 4.3. Let $G=\left(\prod_{i=1}^{n} G_{i}\right)_{H}=\left(\prod_{i=1}^{m} F_{i}\right)_{H}$ be any two central decompositions of $G$ into centrally indecomposable factors. If for each pair of factors $G_{i}$ and $F_{j}$ such that $\bar{G}_{i}$ and $\bar{F}_{j}$ are not exchangeable in the induced direct decompositions of $\bar{G}$ there exist centrally indecomposable subgroups $H \subset U_{i} \subset G_{i}^{\prime}$ and $H \subset V_{j} \subset F_{j}^{\prime}$ such that the decompositions $\bar{G}_{i} \times \bar{U}_{i}=\bar{F}_{j} \times \bar{V}_{j}$ are exchangeable, then any two central decompositions are isomorphic modulo $H$.

Proof. If each pair of factors $G_{i}$ and $F_{j}$ are exchangeable in the induced direct decompositions of $\bar{G}$ then clearly the two central decompositions are isomorphic modulo $H$. Hence we need only consider 
the case when there exist $G_{i}$ and $F_{j}$ such that $\bar{G}_{i}$ and $\bar{F}_{j}$ are not exchangeable in the induced direct decompositions of $\bar{G}$. Thus by hypothesis there exist centrally indecomposable subgroups $U_{i}$ and $V_{j}$ such that the decompositions $\bar{G}_{i} \times \bar{U}_{i}=\bar{F}_{j} \times \bar{V}_{j}$ are exchangeable. Now $\bar{G}_{i}$ and $\bar{F}_{j}$ are not exchangeable in $\bar{G}_{i} \times \bar{G}_{i}^{\prime}=\bar{F}_{j} \times \bar{F}_{j}^{\prime}$. Therefore, by Lemma 4.1, $\bar{G}_{i}$ and $\bar{F}_{j}$ are not exchangeable in $\bar{G}_{i} \times \bar{U}_{i}=$ $\bar{F}_{j} \times \bar{V}_{j}$, whence $\bar{G}_{i}$ and $\bar{V}_{j}$ must be exchangeable. Thus, by Lemma 4.2 , we have

$$
\begin{aligned}
G & =\left(G_{i} \times U_{i} \times G_{i}^{\prime} \cap F_{\jmath}^{\prime}\right)_{H} \\
& =\left(F_{j} \times V_{j} \times G_{i}^{\prime} \cap F_{j}^{\prime}\right)_{H} .
\end{aligned}
$$

Let $G_{i}^{\prime} \cap F_{j}^{\prime}=\left(\prod_{k=1}^{r} N_{k}\right)_{H}$, where the $N_{k}$ 's are centrally indecomposable. Since $U_{i}$ is centrally indecomposable, therefore, considering $G_{i}^{\prime}=\left(\prod_{k=i}^{n} G_{k}\right)_{H}=\left(U_{i} \times \prod_{k-1}^{r} N_{k}\right)_{H}$ and applying induction on $n$, there exists $G_{2}$ such that $\bar{G}_{\lambda} \approx \bar{U}_{i}$ and $\left(\prod_{k=1}^{r} N_{k}\right)_{I I}$ and $\left(\prod_{k \neq i, \lambda}^{n} G_{k}\right)_{H}$ are isomorphic mudulo $H$. In the same way there exists $F_{\mu}$ such that $\bar{F}_{\mu} \approx \bar{V}_{j}$ and $\left(\prod_{k=1}^{r} N_{k}\right)_{H}$ and $\left(\prod_{k \neq j, \mu}^{m} F_{k}\right)_{H}$ are isomorphic modulo $H$. It follows immediately that $m=n$ and $G=\left(\prod_{i=1}^{n} G_{i}\right)_{I I}=\left(\prod_{i=1}^{n} F_{i}\right)_{H}$ are isomorphic modulo $H$.

Lemma 4.4. Let $G=(A \times B)_{H}=(C \times D)_{H}$. Also let $C_{1}=P_{C}(A)$ and $D_{1}=P_{D}(A)$. If $P_{A}\left(C_{1}\right)=A$ then $\left(A \times P_{B}\left(C_{1}\right)\right)_{H}=\left(C_{1} \times D_{1}\right)_{H}$. Moreover $\bar{A}$ and $\bar{C}_{1}$ are exchangeable in $\bar{A} \times \overline{P_{B}\left(C_{1}\right)}=\bar{C}_{1} \times \bar{D}_{1}$.

Proof. Clearly $A \subset\left(C_{1} \times D_{1}\right)_{H I}$. Also $P_{B}\left(C_{1}\right) \subset C_{1} A \subset\left(C_{1} \times D_{1}\right)_{H}$. Therefore $\left(A \times P_{B}\left(C_{1}\right)\right)_{H} \subset\left(C_{1} \times D_{1}\right)_{I I}$. On the other hand,

$$
C_{1} \subset P_{A}\left(C_{1}\right) \cdot P_{B}\left(C_{1}\right) \subset\left(A \times P_{B}\left(C_{1}\right)\right)_{H} .
$$

Moreover, because of Lemma 1 , we can easily see that $P_{B}\left(C_{1}\right)=P_{E}\left(D_{1}\right)$, whence $\left(A \times P_{B}\left(C_{1}\right)\right)_{H}=\left(C_{1} \times D_{1}\right)_{I I}$.

Now $1=\left[D_{1} \cap A, P_{A}\left(C_{1}\right)\right]=\left[D_{1} \cap A, A\right]$. Therefore $D_{1} \cap A=H$. Moreover, since $P_{B}\left(C_{1}\right)=P_{B}\left(D_{1}\right)$, it follows that $C_{1} \cap B \subset P_{B}\left(D_{1}\right)$. Let $b \in C_{1} \cap B$. Then there exists $d \in D$ such that $d=a b$ for some $a \in A$. An easy computation will give

$$
\begin{aligned}
1 & =\left[C_{1}, d\right]=\left[P_{A}\left(C_{1}\right), a\right]\left[P_{B}\left(C_{1}\right), b\right] \\
& =[A, a]\left[P_{B}\left(D_{1}\right), b\right]=[A, a] .
\end{aligned}
$$

Therefore $a \in H$, whence $b \in C_{1} \cap D_{1}=H$. Hence $C_{1} \cap P_{B}\left(C_{1}\right)=H$.

Applying Lemma 2.1, we have $\left[P_{A}\left(C_{1}\right), P_{A}\left(D_{1}\right)\right] \subset H$ and

$$
\left[P_{B}\left(C_{1}\right), P_{B}\left(D_{1}\right)\right] \subset H .
$$

Since $A=P_{A}\left(C_{1}\right)$ and $P_{B}\left(C_{1}\right)=P_{B}\left(D_{1}\right)$ it follows immediately that

$$
\left[\bar{A}, \bar{D}_{1}\right]=\left[\bar{C}_{1}, \overline{P_{B}\left(C_{1}\right)}\right]=1 \text {, }
$$


whence $\bar{A}$ and $\bar{C}_{1}$ are exchangeable in $\bar{A} \times \overline{P_{B}\left(C_{1}\right)}=\bar{C}_{1} \times \bar{D}_{1}$.

If $C$ is centrally indecomposable then by Lemma 2 we must have $C=C_{1}$. Thus we obtain:

CoRollary 4.5. Let $G=(A \times B)_{I I}=(C \times D)_{H}$, where $C$ is centrally indecomposable, and let $C_{1}=P_{C}(A)$ and $D_{1}=P_{D}(A)$. If $P_{A}\left(C_{1}\right)=$ $A$ then $\left(A \times P_{B}(C)\right)_{H}=\left(C \times D_{1}\right)_{H}$. Moreover $\bar{A}$ and $\bar{C}$ are exchangeable in $\bar{A} \times P_{B}(C)=\bar{C} \times \bar{D}_{1}$.

Lemma 4.6. Let $G$ be a p-group of class 2 and let

$$
G=\left(\prod_{i=1}^{n} G_{i}\right)_{H}=\left(\prod_{i=1}^{m} F_{i}\right)_{H}
$$

be two central decompositions of $G$ into centrally indecomposable factors. If for some $i, j$ the abelian group $\bar{G}_{i}$ is of rank $\leqq 3$ then there exist subgroups $U_{i}$ and $B_{j}$ with $H \subset U_{i} \subset G_{i}^{\prime}$ and $H \subset B_{j} \subset F_{j}^{\prime}$ such that the decompositions $\bar{G}_{i} \times \bar{U}_{i}=\bar{F}_{j} \times \bar{B}_{j}$ are exchangeable.

Proof. Let $i=j=1$. Because of Corollary 2.3 we need only prove the case of $G_{1} \neq F_{1}$. Since $\bar{G}_{1}$ is of rank $\leqq 3$ we can let $G_{1}=$ $\left\{s_{1}, s_{2}, s_{3}, H\right\}$. If $\bar{G}_{1}$ is of rank 2 we shall let $s_{3}=1$. Now, by Lemma 1a, there exist $g_{i} \in G_{1}$ and $u_{i} \in G_{1}^{\prime}, i=1,2,3$, such that $g_{i} u_{i} \in F_{1}$ and $g_{i}^{-1} s_{i} u_{i}^{-1} \in F_{1}^{\prime}$. With proper choice of $u_{i}$ we can let $g_{i}=s_{1}^{\alpha_{i} 1} s_{2}^{\alpha_{i}{ }^{2}} s_{3}^{\alpha i 3}$. Since $1=\left[g_{i} u_{i}, g_{i}^{-1} s_{i} u_{i}^{-1}\right]=\left[g_{i}, s_{i}\right]$, writing $\left[s_{1}, s_{2}\right]=h_{1},\left[s_{1}, s_{3}\right]=h_{2}$ and $\left[s_{2}, s_{3}\right]=$ $h_{3}$, we have:

$$
\begin{aligned}
h_{1}^{\alpha_{12}} & =h_{2}^{-\alpha_{13}} \\
h_{1}^{\alpha_{21}} & =h_{3}^{\alpha_{23}} \\
h_{2}^{-\alpha_{31}} & =h_{3}^{\alpha_{32}} .
\end{aligned}
$$

Also $\left[g_{i} u_{i}, g_{j}^{-1} s_{j} u_{j}^{-1}\right]=\left[g_{j} u_{j}, g_{i}^{-1} s_{i} u_{i}^{-1}\right]=1$. Therefore

$$
\left[g_{i}, g_{\jmath}^{-1} s_{j}\right]=\left[u_{i}, u_{\jmath}^{-1}\right]=\left[g_{j}, g_{i}^{-1} s_{i}^{-1}\right]
$$

whence $\left[g_{i}, s_{j}\right]=\left[g_{j}, s_{\imath}\right]^{-1}$. Thus we obtain the following relations:

$$
\begin{aligned}
& h_{1}^{\alpha_{11}-\alpha_{22}}=h_{2}^{\alpha_{23}} h_{3}^{\alpha_{13}} \\
& h_{2}^{\alpha_{11}-\alpha_{33}}=h_{1}^{\alpha_{32}} h_{3}^{-\alpha_{12}} \\
& h_{3}^{\alpha_{22}-\alpha_{33}}=h_{1}^{-\alpha_{31}} h_{2}^{-\alpha_{21}} .
\end{aligned}
$$

Let $A_{1}=\left\{g_{i} u_{i}, H ; i=1,2,3\right\}$ and $B_{1}=\left\{g_{i}^{-1} s_{i} u_{i}^{-1}, H ; i=1,2,3\right\}$. We shall show that at least one of $P_{G_{1}}\left(A_{1}\right)$ and $P_{G_{1}}\left(B_{1}\right)$ coincides with $G_{1}$. If $\bar{G}_{1}$ is of rank 2 then $h_{2}=h_{3}=1$. Thus from relations (1), (2) and (4), we have $h_{1}^{\alpha_{12}}=h_{1}^{\alpha_{21}}=h_{1}^{\alpha_{11}-\alpha_{22}}=1$. This implies that $p \mid \alpha_{12}, \alpha_{2 \mathrm{~s}}$ 
and $\alpha_{11} \equiv \alpha_{22} \bmod p^{k}$ where ord $h_{1}=p^{k}$. Now $g_{i}=s_{1}^{\alpha_{i 1}} s_{2}^{\alpha_{i 2}} \in P_{G_{1}}\left(A_{1}\right)$, $i=1,2$, and $s_{1}^{1-\alpha_{11}} s_{2}^{-\alpha_{12}}, s_{1}^{-\alpha_{21}} s_{2}^{1-\alpha_{22}} \in P_{G_{1}}\left(B_{1}\right)$. Since $p$ does not divide both $\alpha$ and $\alpha-1$ it follows immediately that at least one of $P_{G_{1}}\left(A_{1}\right)$ and $P_{G_{1}}\left(B_{1}\right)$ coincides with $G_{1}$.

If $\bar{G}_{1}$ is of rank 3 we shall show that $p \mid \alpha_{i j}$ for all $i \neq j$ and either $p \mid \alpha_{i i}$ for all $i$ or $p \nmid \alpha_{i i}$ for all $i$. Thus, as in the case of $\bar{G}_{1}$ of rank 2, we have at least one of $P_{G_{1}}\left(A_{1}\right)$ and $P_{G_{1}}\left(B_{1}\right)$ coincides with $G_{1}$.

Suppose $p \nmid \alpha_{13}$. Then by (1) $h_{2} \in\left\{h_{1}\right\}$. Now from (4)

$$
h_{3}^{\alpha_{13}}=h_{1}^{\alpha_{11}-\alpha_{22}} h_{2}^{-\alpha_{23}} \in\left\{h_{1}\right\},
$$

whence $C\left(G_{1}\right)=\left\{h_{1}\right\}$. Since $G_{1}$ is centrally indecomposable, therefore by Theorem 1, this is not possible. Hence $p \mid \alpha_{13}$. In the same way we can show that $p \mid \alpha_{i j}$ for all $i \neq j$.

Suppose now $p \mid \alpha_{11}$ and $p \nmid \alpha_{22}$. Then from (4) we have $h_{1} \in\left\{h_{2}, h_{3}\right\}$. Let $h_{1}=h_{2}^{\mu} h_{3}^{\nu}$. If $p \nmid \alpha_{33}$ then from (5) we have $h_{2}^{\alpha_{11}-\alpha_{33}}=h_{2}^{\mu \alpha_{32}} h_{3}^{\nu} \alpha_{32} h_{3}^{-\alpha_{12}}$. Since $p \mid \alpha_{32}$, it follows that $h_{2} \in\left\{h_{3}\right\}$, whence $C\left(G_{1}\right)$ is cyclic. On the other hand if $p \mid \alpha_{33}$ then using (6) we have $h_{3} \in\left\{h_{2}\right\}$. Hence either $p \mid \alpha_{i i}$ for all $i$ or $p \nmid \alpha_{i i}$ for all $i$.

Now $P_{F_{1}}\left(G_{1}\right)=A_{1}$ and $P_{F_{1}^{\prime}}\left(G_{1}\right)=B_{1}$. If $P_{G_{1}}\left(A_{1}\right)=G_{1}$ then by Corollary 4.5 the lemma follows immediately. If $P_{G_{1}}\left(B_{1}\right)=G_{1}$ then applying Lemmas 4.4 and 4.1 we obtain our required result.

Theorem 4.7. Let $G$ be a p-group of class 2. If

$$
G=\left(\prod_{i=1}^{n} G_{i}\right)_{H}=\left(\prod_{i=1}^{m} F_{i}\right)_{H I}
$$

are two central decompositions of $G$ into centrally indecomposable factors in which the factor group of each factor with respect to the center is abelian of rank $\leqq 3$, then the two central decompositions are isomorphic modulo $H$.

Proof. If for all $i, j, \bar{G}_{i}$ and $\bar{F}_{j}$ are exchangeable in $\prod_{i=1}^{n} \bar{G}_{i}=$ $\prod_{i=1}^{m} \bar{F}_{i}$ then the theorem is immediate. Therefore let $\bar{G}_{i}$ and $\bar{F}_{j}$ be not exchangeable. Since $\bar{G}_{i}$ is abelian of rank $\leqq 3$, therefore by Lemma 4.6, there exist subgroups $H \subset U_{i} \subset G_{i}^{\prime}$ and $H \subset B_{j} \subset F_{j}^{\prime}$ such that the decompositions $\bar{G}_{i} \times \bar{U}_{i}=\bar{F}_{j} \times \bar{B}_{j}$ are exchangeable. Now $\bar{G}_{i}$ and $\bar{F}_{j}$ are of rank at most 3. Thus, by Theorem 3.2, $U_{i}$ and $B_{j}$ are centrally indecomposable. Moreover, by Theorem 4.3, the two central decompositions can be shown isomorphic modulo $H$.

Since a finite nilpotent group is the direct product of its sylow subgroups the following theorems are obvious. 
THEOREM 4.8. If $G$ is a finite nilpotent group such that any two central decompositions of each of its sylow subgroups into centrally indecomposable factors are isomorphic modulo $H_{p}$, where $H_{p}$ is the center of the sylow p-subgroup, then any two central decompositions of $G$ into centrally indecomposable factors are isomorphic modulo $H$.

THEOREM 4.9. If $G$ is a finite nilpotent group of class 2 then any two central decompositions of $G$ into centrally indecomposable factors, in which the factor group of each factor with respect to the center is abelian of rank $\leqq 3$, are isomorphic modulo the center.

Applying Theorem 4.9 and Lemmas 4.6 and 4.2 it is not difficult to obtain:

THEOREM 4.10. If $G$ is a finite nilpotent group such that the factor group of each of the sylow subgroups with respect to its center is abelian of rank $\leqq 7$, then any two central decompositions of $G$ into centrally indecomposable factors are isomorphic modulo the center.

Thus we note that any central decompositions of a $p$-group of class 2 generated by seven $H$-generators into centrally indecomposable factors are isomorphic modulo the center. In $\S 6$ we have an example of a p-group of class 2 generated by nine $H$-generators which admits central decompositions into centrally indecomposable factors that are not isomorphic modulo the center. The question whether there exist a nilpotent group of class 2 generated by eight $H$-generators admitting central decompositions into centrally indecomposable factors that are not isomorphic modulo the center is still undecided.

5. In this section we shall show that if a $p$-group $G$ admits a central decomposition with each of its factors generated by two $H$ generators then any two central decompositions of $G$ into centrally indecomposable factors are isomorphic modulo the center.

LEMMA 5.1. Let $G=\left(\prod_{i=1}^{n} G_{i}\right)_{H}=\left(\prod_{i=1}^{m} F_{i}\right)_{H}$. If there exists $F_{i}$ such that $\bar{F}_{i}$ is exchangeable with $\bar{G}_{j}$ for all $j$ in the induced direct decompositions of $\bar{G}$ then $G$ is nilpotent of class 2.

Proof. Let $F_{1}$ be such that $\bar{F}_{1}$ is exchangeable with every $\bar{G}_{j}$ in the induced direct decompositions of $\bar{G}$. This implies that $\bar{F}_{1} \theta_{j}=\bar{G}_{j}$ for all $j$, where $\theta_{j}$ is the $\bar{G}_{j}$-decomposition operator. Moreover $1=$ $\left[\bar{F}_{1}, \bar{G}_{j}\right]=\left[\bar{F}_{1} \theta_{j}, \bar{G}_{j}\right]$ for all $j$, whence $G_{j}$ is nilpotent of class 2 for all $j$. Therefore $G$ is nilpotent of class 2 . 
LEMMA 5.2. Let $G$ be a p-group and let $G=\left(\prod_{i=1}^{n} G_{i}\right)_{H}=\left(\prod_{i=1}^{m} F_{i}\right)_{H}$ where each $G_{i}$ is generated by two $H$-generators. If there exists an $F_{i}$ such that $\bar{F}_{i}$ is exchangeable with $\bar{G}_{j}$ for all $j$ in $\prod_{i=1}^{n} \bar{G}_{i}=\prod_{i=1}^{m} \bar{F}_{i}$, then $C(G)$ is cyclic.

Proof. Let $F_{1}$ be such a factor. Then by Lemma $5.1 \mathrm{G}$ is nilpotent of class 2. Thus for $G_{i}=\left\{a_{i}, b_{i}, H\right\}$ we have $\left[a_{i}, b_{i}\right]=h_{i} \in$ $Z(G)=H$. Now $\bar{F}_{1}$ exchangeable with $\bar{G}_{i}$ implies that $\bar{F}_{1}$ is of rank 2. Moreover if $F_{1}=\{a, b, H\}$ then $\left\{\bar{a} \theta_{i}, \bar{b} \theta_{i}\right\}=\bar{G}_{i}$, where $\theta_{i}$ is the $\bar{G}_{i^{-}}$ decomposition operator. Thus without loss of generality we can let $a=a_{1} a_{2}, \cdots, a_{n}$ and $b=b_{1} b_{2}, \cdots, b_{n}$. Now $a_{i}=x_{i} y_{i}$ for some $x_{i} \in F_{1}$ and $y_{i} \in F_{1}^{\prime}$. Let $x_{i}=a^{\alpha} b^{\beta}{ }^{\beta} k_{i}, k_{i} \in H$. Then $y_{i}=a^{-\alpha_{i}} b^{-\beta_{i}} a_{i} k_{i}^{\prime}, k_{i}^{\prime} \in H$. But $\left[b, y_{i}\right]=1$. This implies that

$$
h_{1}^{\alpha_{i}} \cdots h_{i-1}^{\alpha_{i}} h_{i}^{\alpha_{i-2}} h_{i+1}^{\alpha_{i}} \cdots h_{n}^{\alpha_{i}}=1
$$

for all $i$.

Let $p^{r}$ be the highest order of the $h_{i}$ 's. Then for each pair of $\alpha_{i}$ and $\alpha_{j}, i \neq j$, at least one is a multiple of the other modulo $p^{r}$. Let $\alpha_{j} \equiv k \alpha_{i} \bmod p^{r}$ (say). This implies that,

$$
1=h_{1}^{k \alpha_{i}} \cdots h_{n}^{k \alpha_{\imath}} h_{\imath}^{-k}=h_{1}^{\alpha}{ }_{\jmath} \ldots h_{n}^{\alpha} j h_{\imath}^{-k} .
$$

Therefore $h_{i}^{k}=h_{1}^{\alpha}{ }^{\alpha} \cdots h_{n}^{\alpha_{j}}$. But $h_{1}^{\alpha_{j}} \cdots h_{n}^{\alpha_{j}}=h_{j}$. Hence $h_{j} \in H_{i}=\left\{h_{i}\right\}_{\text {. }}$ Thus for each pair of $h_{i}$ and $h_{j}$ either $H_{i} \leqq H_{j}$ or $H_{j} \leqq H_{i}$, whence $C(G)$ is cyclic.

THEOREM 5.3. If $G$ is a p-group admitting a central decomposition into centrally indecomposable factors with each factor generated by two H-generators, then any two central decompositions of $G$ into centrally indecomposable factors are isomorphic modulo the center.

Proof. Let $G=\left(\prod_{i=1}^{n} G_{i}\right)_{H}=\left(\prod_{i=1}^{m} F_{i}\right)_{H}$, where $G_{i}$ is generated by two $H$-generators for all $i$. If every $\bar{G}_{i}$ is exchangeable with every $\bar{F}_{j}$ in $\prod_{i=1}^{n} \bar{G}_{i}=\prod_{i=1}^{m} \bar{F}_{i}$ then our theorem is true. Therefore we shall assume that there exists a pair of $G_{i}$ and $F_{j}$ such that $\bar{G}_{i}$ and $\bar{F}_{j}$ are not exchangeable. Let $i=j=1$. Let $G_{1}=\left\{s_{1}, s_{2}, H\right)$. Then, by Lemma 1a, there exist elements $a_{i}=g_{2} u_{i} \in F_{1}$ and $b_{i}=g_{i}^{-1} s_{i} u_{i}^{-1} \in F_{1}^{\prime \prime}$, $g_{i} \in G_{1}$ and $u_{i} \in G_{1}^{\prime}, i=1,2$. Let $U=\left\{u_{1}, u_{2}, H\right\}, A=\left\{a_{1}, a_{2}, H\right\}$ and $B=\left\{b_{1}, b_{2}, H\right\}$. It is easy to see that $C=\left(G_{1} \times U\right)_{H}=(A \times B)_{H}$. We shall show that $\bar{G}_{1}$ and $\bar{B}$ are exchangeable in $\bar{G}_{1} \times \bar{U}=\bar{A} \times \bar{B}$. If $C$ is nilpotent of class 2 , then, by Lemma $4.6, \bar{G}_{1}$ is exchangeable with either $\bar{A}$ or $\bar{B}$. But, by Corollary $4.5, \bar{G}_{1}$ and $\bar{A}$ exchangeable will imply $\bar{G}_{1}$ and $\bar{F}_{1}$ exchangeable in $\bar{G}_{1} \times \bar{U}=\bar{F}_{1} \times \bar{B}$, whence by Lemma 4.1 contradicting the inexchangeability of $\bar{G}_{1}$ and $\bar{F}_{1}$ in

$$
\prod_{i=1}^{n} \bar{G}_{i}=\prod_{i=1}^{m} \bar{F}_{i} .
$$


Therefore $\bar{G}_{1}$ and $\bar{B}$ are exchangeable in $\bar{G}_{1} \times \bar{U}=\bar{A} \times \bar{B}$. If $C$ is not nilpotent of class 2 , then, since $U$ is nilpotent of class 2 , we must have $G_{1}$ not nilpotent of class 2 . This means that $\bar{G}_{1}$ is directly indecomposable. Therefore by Remak-Krull-Schmidt theorem $\bar{G}_{1}$ must be exchangeable with either $\bar{A}$ or $\bar{B}$. Again as it is in the case of $C$ being nilpotent of class 2 the exchangeability of $\bar{G}_{1}$ and $\bar{A}$ is impermissible. Hence $\bar{G}_{1}$ and $\bar{B}$ are exchangeable. Thus by Lemma 4.1, $\bar{G}_{1}$ and $\bar{B}$ are exchangeable in $\bar{G}_{1} \times P_{G_{1}^{\prime}}\left(F_{1}\right)=\bar{F}_{1} \times \bar{B}$.

We shall now show that $P_{G_{1}^{\prime}}\left(F_{1}\right)=V$ is either centrally indecomposable or $G$ is nilpotent of class 2 with cyclic $C(G)$. Let

$$
V=\left(\prod_{i=1}^{r} V_{i}\right)_{H}
$$

where each $V_{i}$ is centrally indecomposable.

Case 1. $\quad V \neq G_{1}^{\prime}$. Then by induction on $n$ we must have $r<n-1$. Thus $\left(G_{1} \times V_{1} \times \cdots \times V_{r}\right)_{H}$ has $r+1<n$ factors. Hence again by induction this decomposition is isomorphic modulo $H$ to $\left(F_{1} \times B\right)_{H}$, whence $r=1$. Therefore $V$ is centrally indecomposable.

Case 2. $V=G_{1}^{\prime}$. Since $\bar{F}_{1}$ and $\bar{V}$ are exchangeable, therefore $\bar{F}_{1}$ and $\bar{V}_{i}$ are not exchangeable for all $i$, unless $r=1$, in which case $V$ is centrally indecomposable. Thus as in the first part of the proof, for each $i$ there exist $W_{i} \subseteq V_{i}^{\prime}$ and $B_{i}^{*} \subseteq F_{\perp}^{\prime}$ such that $\left(V_{i} \times W_{i}\right)_{H}=$ $\left(F_{1} \times B_{i}^{*}\right)_{H}$ with $\bar{V}_{i}$ and $\bar{B}_{i}^{*}$ exchangeable in $\bar{V}_{i} \times \bar{W}_{i}=\bar{F}_{1} \times \bar{B}_{i}^{*}$. Now suppose $W_{i} \neq V_{i}^{\prime}$ for some $i$. Since by induction the decompositions $G_{1}^{\prime}=\left(\prod_{i=2}^{n} G_{i}\right)_{H}=\left(\prod_{i=1}^{r} V_{i}\right)_{H}$ are isomorphic modulo $H$, therefore, applying the argument of Case 1 , we have $r=1$, whence $V$ is centrally indecomposable. Thus we need only consider the case of $W_{i}=V_{i}^{\prime}$ for all $i$. This means,

$$
G=\left(V_{i} \times W_{i}\right)_{H}=\left(F_{1} \times B_{i}^{*}\right)_{H}=\left(F_{1} \times B\right)_{H}
$$

for all $i$.

Thus by Corollary 2.3, $B_{i}^{*}=B$ for all $i$. Thus $\bar{V}_{i}$ and $\bar{B}$ are exchangeable in $\bar{V}_{i} \times \bar{V}_{i}^{\prime}=\bar{F}_{1} \times \bar{B}$ for all $i$. Since $\bar{G}_{1}$ and $\bar{B}$ are exchangeable in $\bar{G}_{1} \times \bar{G}_{1}^{\prime}=\bar{F}_{1} \times \bar{B}$, therefore, by Lemmas 5.1 and 5.2, $G$ must be nilpotent of class 2 with cyclic $C(G)$.

Now, if $V=P_{G^{\prime}}\left(F_{1}\right)$ is centrally indecomposable then by induction on $n$ and Theorem 4.3 the decompositions $\left(\prod_{i=1}^{n} G_{i}\right)_{H}=\left(\prod_{i=1}^{m} F_{i}\right)_{H}$ are isomorphic modulo $H$. If $G$ is nilpotent of class 2 with cyclic $C(G)$ then by Theorem 1 we reach the same conclusion.

COROLLARY 5.4. If $G$ is a finite nilpotent group with each of 
its sylow subgroups generated by at most four $H_{p}$-generators, $H_{p}$ being the center of the sylow p-subgroup, then any two central decompositions into centrally indecomposable factors are isomorphic modulo the center.

6. We shall now give an example of a $p$-group of class 2 admitting two central decompositions into centrally indecomposable factors which are not isomorphic modulo the center.

Let $G$ be generated by $a_{i}, b_{i}, c_{i}, i=1,2,3$, satisfying the following relations:

$$
\begin{gathered}
a_{i}^{2}=b_{i}^{2}=c_{i}^{2}=1 \\
{\left[a_{i}, b_{j}\right]=\left[a_{i}, c_{j}\right]=\left[b_{i}, c_{j}\right]=1} \\
{\left[a_{i}, a_{j}\right]=\left[b_{i}, b_{j}\right]=\left[c_{i}, c_{j}\right] \in Z(G)} \\
{\left[a_{i}, a_{j}\right]^{2}=1 .}
\end{gathered}
$$

Thus $G$ is a nilpotent 2-group with center $Z(G)=H=\left\{h_{1}, h_{2}, h_{3}\right\}$ where $h_{1}=\left[a_{1}, a_{2}\right], h_{2}=\left[a_{1}, a_{3}\right]$ and $h_{3}=\left[a_{2}, a_{3}\right]$. Now let $A=\left\{a_{1}, a_{2}, a_{3}\right\}, B=$ $\left\{b_{1}, b_{2}, b_{3}\right\}$ and $C=\left\{c_{1}, c_{2}, c_{3}\right\}$. Then $G=(A \times B \times C)_{H}$. On the other hand if we let

$$
F_{1}=\left\{a_{1} b_{1} c_{1}, a_{2} b_{2} c_{2}, a_{3} b_{3} c_{3}\right\}
$$

and

$$
F_{2}=\left\{a_{1} c_{1}, a_{2} c_{2}, a_{3} c_{3}, b_{1} c_{1}, b_{2} c_{2}, b_{3} c_{3}\right\}
$$

then $G=\left(F_{1} \times F_{2}\right)_{H}$. By Theorem 3.2, $A, B, C$, and $F_{1}$ are all centrally indecomposable. We need to show that $F_{2}$ is centrally indecomposable. To prove this we shall first show that the subgroup $T=$ $(A \times B)_{H}$ has a unique central decomposition into centrally indecomposable factors.

Suppose $T$ has another central decomposition into centrally indecomposable factor. Then by Theorem 4.10, $T=(D \times E)_{H}$ is isomorphic modulo $H$ to $T=(A \times B)_{H}$. Now, by Lemma 1a, there exist elements $d_{i}=g_{i} u_{i} \in D$ and $e_{i}=g_{i}^{-1} a_{i} u_{i}^{-1} \in E$, where $g_{i}=a_{1}^{\alpha i_{1}} a_{2}^{\alpha_{i 1}} a_{3}^{\alpha_{i 3}}$ and $u_{i} \in B$. Computing the same way as it is in Lemma 4.6, we obtain the relations (1)-(6) of $\S 4$. But in $G, h_{i}^{\alpha}=h_{j}^{\beta}$ for $i \neq j$ if and only if $\alpha \equiv$ $\beta \equiv 0 \bmod 2$. Therefore $\alpha_{i j} \equiv 0 \bmod 2$ for $i \neq j$ and $\alpha_{11} \equiv \alpha_{22} \equiv \alpha_{33}$ $\bmod 2$. Thus if $\alpha_{11} \equiv 1 \bmod 2$ then $A=D$ and if $\alpha_{11} \equiv 0 \bmod 2$ then $A=E$, whence, by Corollary 2.3, $T$ has a unique decomposition into centrally indecomposable factors.

Now suppose $F_{2}=(U \times V)_{H}$.

Case 1. There exists one of $\bar{U}$ and $\bar{V}$ which is not exchangeable 
with one of $\bar{A}, \bar{B}$ and $\bar{C}$. Let $\bar{A}$ and $\bar{U}$ (say) be not exchangeable. Then, by Lemma 4.6, there exist subgroups $H \subset Q \subset A^{\prime}$ and $H \subset R \subset U^{\prime}$ such that $(A \times Q)_{H}=(U \times R)_{H}$ with $\bar{A}$ and $\bar{R}$ exchangeable in

$$
\bar{A} \times \bar{Q}=\bar{U} \times \bar{R} .
$$

Thus,

$$
\begin{aligned}
G & =\left(A \times Q \times A^{\prime} \cap U^{\prime}\right)_{H} \\
& =\left(U \times R \times A^{\prime} \cap U^{\prime}\right)_{H} .
\end{aligned}
$$

But $\left(Q \times A^{\prime} \cap U^{\prime}\right)_{H}=(B \times C)_{H}$ which has a unique central decomposition into centrally indecomposable factors. Therefore $Q=B$ or $C$. Let $Q=B$ (say). This implies that $U=B$ and $A=R$, whence $B \subset F_{2}$. But it is easy to see that $B \cap F_{2}=H$. Hence this case does not exist.

Case 2. Every factor of $\bar{G}=\bar{A} \times \bar{B} \times \bar{C}$ is exchangeable with every factor of $\bar{G}=\bar{F}_{1} \times \bar{U} \times \bar{V}$. Now the exchangeability of $\bar{U}$ and $\bar{C}$ implies that there exists $u=w_{1}\left(a_{i}\right) w_{2}\left(b_{i}\right) c_{1} \in U$ where $w_{1}\left(a_{i}\right)$ and $w_{2}\left(b_{i}\right)$ are words on $a_{i}$ 's and $b_{i}$ 's respectively. In fact for convenience we shall regard $w_{1}$ and $w_{2}$ as functions. Since $\left[a_{1} b_{1} c_{1}, u\right]=1$ we have either

$$
\left[a_{1}, w_{1}\left(a_{i}\right)\right]=\left[b_{1}, w_{2}\left(b_{i}\right)\right]^{-1}=\left[b_{1}, w_{2}\left(b_{i}\right)\right] \neq 1
$$

or

$$
\left[a_{1}, w_{1}\left(a_{i}\right)\right]=\left[b_{1}, w_{2}\left(b_{i}\right)\right]=1 .
$$

Suppose (1) is true. Then since $A$ and $B$ are isomorphic under the map $\psi: a_{i} \rightarrow b_{i}$ with $\psi$ fixing $H$, therefore, $\left[a_{1}, w_{1}\left(a_{i}\right)\right]=\left[b_{1}, w_{2}\left(b_{i}\right)\right] \mathrm{im}$ plies that $\left[b_{1}, w_{1}\left(b_{i}\right)\right]=\left[b_{1}, w_{2}\left(b_{i}\right)\right]$, whence $w_{1}\left(b_{i}\right)=w_{2}\left(b_{i}\right) h$ for some $h \in H$. Thus $u=w_{1}\left(a_{i}\right) w_{1}\left(b_{i}\right) c_{1} h^{-1}$. Now $\bar{G}$ is the free abelian product of the cyclic subgroups $\left\{\bar{a}_{i}\right\},\left\{\bar{b}_{i}\right\},\left\{\bar{c}_{i}\right\}, i=1,2,3$. Clearly the length of $\bar{u}$ on $\bar{a}_{i}, \bar{b}_{i}, \bar{c}_{i}, i=1,2,3$ is odd. But it is not difficult to see that every element of $\bar{F}_{2}$ is of even length. Hence (1) cannot be true.

Now, if (2) is true, then $w_{1}\left(a_{i}\right) \in Z_{A}\left(a_{1}\right)=\left\{a_{1}, H\right\}$ and

$$
w_{2}\left(b_{i}\right) \in Z_{B}\left(b_{1}\right)=\left\{b_{1}, H\right\} .
$$

Thus $u$ is of the form $a_{1} b_{1} c_{1} h, a_{1} c_{1} h, b_{1} c_{1} h$ or $c_{1} h$ for some $h \in H$. Since $a_{1} b_{1} c_{1} h, c_{1} h \in F_{2}$, therefore $u$ can be assumed to be either $a_{1} c_{1}$ or $b_{1} c_{1}$. In the same way there exist $v \in V$ such that $v$ can be assumed to be either $a_{1} c_{1}$ or $b_{1} c_{1}$. Since $U \cap V=H$, therefore, if $a_{1} c_{1} \in U$ then $b_{1} c_{1} \in V$ or vice versa. Now $F_{2}$ is generated by $a_{1} c_{1}, a_{2} c_{2}, a_{3} c_{3}, b_{1} c_{1}, b_{2} c_{2}, b_{3} c_{3}$, whence $U$ or $V$ is generated by a proper choice of three of these elements. But any such choice will not satisfy $[U, V]=1$. Hence $F_{2}$ is centrally indecomposable. 


\section{REFERENCES}

1. D. Gorenstein, Finite groups, Harper and Row, New York, 1968.

2. P. Hall, Finite-by-nilpotent groups, Proc. Comb. Phil. Soc. 52 (1956), 611-616.

3. I. Kaplansky, Infinite Abelian groups, The University of Michigan Press, 1954.

4. B. H. Neumann and H. Neumann, A remark on generalized free products, J. London Math. Soc. 25 (1950), 202-204.

5. C. Y. Tang, On uniqueness of generalized direct decompositions, Pacific J. Math. 23 (1967), 171-182

Received July 14, 1969. This work was partly supported by a grant from the Canadian National Research Council No. A-4064. Part of the work was done when the author was a fellow of the Summer Research Institute at the University of British Columbia.

UNIVERSITY OF WATERLOO

Waterloo, Ontario, Canada 



\section{PACIFIC JOURNAL OF MATHEMATICS}

\section{EDITORS}

\author{
H. SAMELSON \\ Stanford University \\ Stanford, California 94305 \\ Richard Pierce \\ University of Washington \\ Seattle, Washington 98105
}

J. DUGUNDJI

Department of Mathematics

University of Southern California

Los Angeles, California 90007

RICHARD ARENS

University of California

Los Angeles, California 90024

\section{ASSOCIATE EDITORS}
E. F. BECKENBACH
B. H. NEUMaNN
F. WOLE
K. YosHIDA

\section{SUPPORTING INSTITUTIONS}

\author{
UNIVERSITY OF BRITISH COLUMBIA \\ CALIFORNIA INSTITUTE OF TECHNOLOGY \\ UNIVERSITY OF CALIFORNIA \\ MONTANA STATE UNIVERSITY \\ UNIVERSITY OF NEVADA \\ NEW MEXICO STATE UNIVERSITY \\ OREGON STATE UNIVERSITY \\ UNIVERSITY OF OREGON \\ OSAKA UNIVERSITY \\ UNIVERSITY OF SOUTHERN CALIFORNIA
}

\author{
STANFORD UNIVERSITY \\ UNIVERSITY OF TOKYO \\ UNIVERSITY OF UTAH \\ WASHINGTON STATE UNIVERSITY \\ UNIVERSITY OF WASHINGTON

$* * *{ }^{*} *{ }^{*}$
AMERICAN MATHEMATICAL SOCIETY
CHEVRON RESEARCH CORPORATION
TRW SYSTEMS \\ NAVAL WEAPONS CENTER
}

The Supporting Institutions listed above contribute to the cost of publication of this Journal. but they are not owners or publishers and have no responsibility for its content or policies.

Mathematical papers intended for publication in the Pacific Journal of Mathematics should be in typed form or offset-reproduced, (not dittoed), double spaced with large margins. Underline Greek letters in red, German in green, and script in blue. The first paragraph or two must be capable of being used separately as a synopsis of the entire paper. The editorial "we" must not be used in the synopsis, and items of the bibliography should not be cited there unless absolutely necessary, in which case they must be identified by author and Journal, rather than by item number. Manuscripts, in duplicate if possible, may be sent to any one of the four editors. Please classify according to the scheme of Math. Rev. 36, 1539-1546. All other communications to the editors should be addressed to the managing editor, Richard Arens, University of California, Los Angeles, California, 90024.

50 reprints are provided free for each article; additional copies may be obtained at cost in multiples of 50 .

The Pacific Journal of Mathematics is published monthly. Effective with Volume 16 the price per volume (3 numbers) is $\$ 8.00$; single issues, $\$ 3.00$. Special price for current issues to individual faculty members of supporting institutions and to individual members of the American Mathematical Society: $\$ 4.00$ per volume; single issues $\$ 1.50$. Back numbers are available.

Subscriptions, orders for back numbers, and changes of address should be sent to Pacific Journal of Mathematics, 103 Highland Boulevard, Berkeley, California, 94708.

PUBLISHED BY PACIFIC JOURNAL OF MATHEMATICS, A NON-PROFIT CORPORATION

Printed at Kokusai Bunken Insatsusha (Internatıonal Academic Printing Co., Ltd.), 7-17, Fujimi 2-chome, Chiyoda-ku, Tokyo, Japan. 


\section{Pacific Journal of Mathematics}

\section{Vol. 33, No. 3 \\ May, 1970}

Charles A. Akemann, Approximate units and maximal abelian

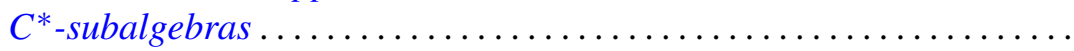

Gail Atneosen, Wild points of cellular arcs in 2-complexes in $E^{3}$ and cellular hulls

John Logan Bryant and De Witt Sumners, On embeddings of 1-dimensional

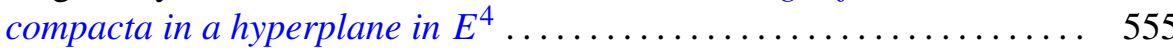

H. P. Dikshit, On a class of Nörlund means and Fourier series.......... 559

Nancy Dykes, Generalizations of realcompact spaces .............. 571

Hector O. Fattorini, Extension and behavior at infinity of solutions of certain linear operational differential equations .................. 583

Neal David Glassman, Cohomology of nonassociative algebras .......... 617

Neal Hart, Ulm's theorem for Abelian groups modulo bounded groups . . . . 635

Don Barker Hinton, Continuous spectra of second-order differential

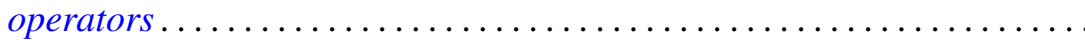

Donald Gordon James, On Witt's theorem for unimodular quadratic forms. II ............................................. 645

Melvin F. Janowitz, Principal multiplicative lattices................ 653

James Edgar Keesling, On the equivalence of normality and compactness in hyperspaces .................................... 657

Adalbert Kerber, Zu einer Arbeit von J. L. Berggren über ambivalente Gruppen

Keizō Kikuchi, Various $m$-representative domains in several complex variables

Jack W. Macki and James Stephen Muldowney, The asymptotic behaviour of solutions to linear systems of ordinary differential equations ....

Andy R. Magid, Locally Galois algebras . . . .

T. S. Ravisankar, On differentiably simple algebras......

Joseph Gail Stampfli, The norm of a derivation ..

Francis C.Y. Tang, On uniqueness of central decompositions of groups ...

749

Robert Charles Thompson, Some matrix factorization theorems. I .

763

Robert Charles Thompson, Some matrix factorization theorems. II . . 